DOI: 10.12957/demetra.2015.15949

\title{
Avaliação antropométrica de idosos atendidos no Ambulatório de Nutrição do Centro de Referência em Assistência à Saúde do Idoso da Universidade Federal Fluminense, no município de Niterói-RJ
}

\author{
Anthropometric assessment in elderly patients receiving nutritional consultation in a reference \\ center in assistance of elderly health at Universidade Federal Fluminense, in Niterói-RJ, Brazil
}

Ana Luiza Sant'Anna da Costa Silva'

Beatriz dos Santos da Silva ${ }^{2}$

Joana Maia Brandão ${ }^{2}$

Sergio Girão Barroso ${ }^{\top}$

Gabrielle de Souza Rocha'

1 Universidade Federal Fluminense, Faculdade de Nutrição Emilia de Jesus Ferreiro. Niterói-RJ, Brasil.

${ }^{2}$ Universidade Federal Fluminense, Residência Multiprofissional. Niterói-RJ, Brasil.

Correspondência / Correspondence Gabrielle de Souza Rocha

E-mail: profgabrielle.rocha2@globo.com

\section{Resumo}

Objetivo: Descrever o perfil antropométrico e a composição corporal de idosos atendidos no Ambulatório de Nutrição do Centro de Referência em Assistência à Saúde do Idoso da Universidade Federal Fluminense. Metodologia: Trata-se de um estudo transversal, realizado com 52 indivíduos com 60 anos ou mais atendidos no ambulatório de nutrição. As medidas antropométricas foram realizadas segundo as diretrizes da International Society for the Advancement of Kineanthropometry, considerando as seguintes variáveis: massa corporal, estatura, dobras cutâneas biciptal, triciptal, subescapular, supra-ilíaca, percentual de gordura corporal a partir do somatório das quatro dobras cutâneas, circunferência da cintura e circunferência do braço. As análises estatísticas foram realizadas utilizando o software Graph Pad inStat ${ }^{\circledR}$, versão 3.1, considerando-se significativo p $<0,05$. Resultados: Verificou-se prevalência de sobrepeso (43,5\% da amostra), adequação da circunferência do braço, circunferência muscular do braço e dobra cutânea triciptal. A maior parte das mulheres $(67,6 \%)$ apresentava risco elevado para doenças cardiovasculares segundo a circunferência abdominal. Observou-se percentual de gordura corporal elevado em ambos os sexos (89,2\% e 66,7\%, feminino e masculino, respectivamente). Conclusão: Observou-se a presença de inadequações do estado nutricional da população em estudo de acordo com os parâmetros antropométricos índice de massa corporal, circunferência abdominal e percentual de gordura pelo somatório de dobras.

Palavras-chave: Envelhecimento. Antropometria. Avaliação Nutricional. Estado Nutricional. Doença Crônica. 


\section{Abstract}

Objective: To describe the body and anthropometric composition of elderly assisted in the Nutrition Clinic of the Reference Center of Elderly's Health, Universidade Federal Fluminense, Niteroi city, Brazil. Methodology: This is a cross-sectional study with 52 subjects aged 60 years or older treated at the nutrition clinic. Anthropometric measurements were performed according to the guidelines of the International Society for the Advancement of Kineanthropometry considering the following variables: body weight, height, skinfold biceps, triceps, subscapular, supra-iliac, body fat percentage from the sum of the four folds skin, waist circumference and arm circumference. Statistical analyzes were performed using Graph Pad Instat ${ }^{\circledR}$ software, version 3.1, considering significant a $\mathrm{p}<0.05$. Results: There was prevalence of overweight ( $43.5 \%$ of the sample), adequacy of the arm circumference, arm muscle circumference and triceps skinfold. Most women (67.6\%) had high risk for cardiovascular disease according to waist circumference. There was high percentage of body fat in both sexes $(89.2 \%$ and $66.7 \%$ in female and male, respectively). Conclusion: We observed the presence of inadequacies of the nutritional status of the population under study according to anthropometric parameters - body mass index, waist circumference, and percent body fat by skinfold sum.

Key words: Aging. Anthropometry. Nutrition Assessment. Nutritional Status. Chronic Disease.

\section{Introdução}

O envelhecimento é um processo universal que caracteriza uma etapa da vida permeada por mudanças sociais, psíquicas, ambientais e biológicas, que compõem o desenvolvimento normal e integral do homem. ${ }^{1}$ Já o envelhecimento da população idosa brasileira é um fenômeno recente e está fortemente atrelado à melhoria da qualidade de vida, à tendência de diminuição do crescimento populacional, ao melhor controle dos agravos à saúde e intensa urbanização desse grupo etário. ${ }^{2}$ No município de Niterói, há 83.536 idosos, sendo 51.024 do sexo feminino e 32.512 do sexo masculino, segundo dados do Instituto Brasileiro de Geografia e Estatística (IBGE). ${ }^{3}$

De acordo com Panziera et al., ${ }^{4}$ o processo de envelhecimento acarreta mudanças fisiológicas que interferem no estado nutricional e contribuem para os idosos constituírem o grupo mais mal-nutrido. 
A avaliação nutricional do idoso deve conter anamnese detalhada das alterações fisiológicas apresentadas pelo individuo. A avaliação do estado nutricional abrange vários métodos e técnicas, dentre os quais se destaca a antropometria. Este método permite avaliar alterações nos compartimentos corporais decorrentes do processo de envelhecimento, como a diminuição da estatura e massa livre de gordura, e modificação na redistribuição de gordura corporal, onde há aumento do tecido adiposo central e diminuição do tecido adiposo periférico. ${ }^{5}$

Utilizando dados nutricionais da clientela atendida nos serviços de saúde, ou dados de base populacional, é possível traçar o perfil da população-alvo. O conhecimento do perfil antropométrico de uma população pode funcionar como fator de alerta para a tomada de decisões das autoridades de saúde, órgãos públicos, bem como para as instituições assistenciais cujo foco seja o idoso, assim como do Centro de Referência em Assistência à Saúde do Idoso (CRASI), no município de Niterói, no sentido de criar programas e ações preventivas que possam minimizar, no médio e longo prazos, as tendências e fatores determinantes próprios da idade. ${ }^{6}$

O presente estudo tem por objetivo traçar o perfil antropométrico e descrever a composição corporal de idosos atendidos no ambulatório de nutrição do CRASI da Universidade Federal Fluminense (UFF), no município de Niterói-RJ.

\section{Metodologia}

\section{Amostra}

Trata-se de estudo transversal com coleta de dados realizada no período de julho a setembro de 2013. Foram avaliados 52 idosos, com 60 anos ou mais, de ambos os sexos, atendidos no ambulatório de nutrição do CRASI/UFF. Como critérios de exclusão, foram considerados aqueles sem condições físicas para avaliação nutricional.

O convite aos indivíduos foi feito verbalmente e, após esclarecimentos sobre a metodologia, risco e objetivos do trabalho, os mesmos ou seus responsáveis assinaram um Termo de Consentimento Livre e Esclarecido.

O projeto de estudo foi submetido ao Comitê de Ética e Pesquisa da Faculdade de Medicina da Universidade Federal Fluminense, tendo sido aprovado sob o protocolo número 0084.0.258.0000713343513.2.0000.5243, no ano de 2013, por estar de acordo com a Resolução n 466/2012 do Conselho Nacional de Saúde. 


\section{Avaliação antropométrica}

As medições antropométricas foram realizadas segundo as diretrizes da International Society for the Advancement of Kineanthropometry (ISAK). ${ }^{7}$ Foram determinadas as seguintes variáveis antropométricas: massa corporal, estatura, dobras cutâneas biciptal, triciptal, subescapular e supra-ilíaca, percentual de gordura corporal a partir do somatório das quatro dobras cutâneas, circunferência da cintura e circunferência do braço. As medidas foram triplicadas por avaliadores experientes, utilizando como resultado a média aritmética das mesmas.

A massa corporal foi obtida por meio de uma balança digital da marca Filizola®, com capacidade de 150 quilogramas (Kg) e precisão de 100 gramas (g); a estatura, em metros (m), por meio de um estadiômetro vertical fixo à balança; a espessura das dobras cutâneas foi aferida, em triplicata, do lado direito do indivíduo, utilizando um adipômetro científico de marca Cescorf® com precisão de um milímetro $(\mathrm{mm})$ e os perímetros com o auxílio de uma fita antropométrica Cescorf® com precisão de $1 \mathrm{~mm}$.

O índice de massa corporal (IMC) foi calculado através da razão entre a massa corporal (Kg) e o quadrado da estatura $(\mathrm{m})$. O IMC $\left(\mathrm{kg} / \mathrm{m}^{2}\right)$ foi classificado segundo os pontos de corte propostos pela World Health Organization (WHO ${ }^{8}$ A circunferência da abdominal (CAb) foi classificada segundo o ponto de corte estabelecido para população de acordo com Alberti et al. ${ }^{9}$

A estimativa da gordura corporal total foi realizada pelo modelo bicompartimental, utilizando-se o somatório das dobras cutâneas triciptal, biciptal, suprailíaca e subescapular, em milímetros. ${ }^{10} \mathrm{~A}$ partir do valor encontrado no cálculo do somatório de dobras, o percentual de gordura corporal total para homens e mulheres foi estimado de acordo com a faixa etária. ${ }^{11}$

\section{Análise estatística}

As análises estatísticas foram realizadas utilizando o software Graph Pad inStat ${ }^{\circledR}$, versão 3.1. Para a descrição das variáveis contínuas, utilizou-se a média aritmética, com seu respectivo desviopadrão (DP), e para as variáveis categóricas, a frequência absoluta (N) e o percentual (\%). Para avaliar a associação entre os sexos e as variáveis antropométricas, empregou-se o teste Exato de Fisher, considerando significativo quando $\mathrm{p}<0,05$. 


\section{Resultados}

Foram entrevistados 52 idosos, mas 11,5\% $(\mathrm{n}=6)$ foram excluídos da amostra, pois não apresentavam condições físicas para avaliação nutricional. A amostra foi constituída por 46 idosos, sendo 80,4\% (n=37) do sexo feminino e 19,6\% (n=9) do sexo masculino. Verificou-se que a idade dos idosos estudados variou entre 60 e 90 anos, e a média da idade \pm desvio padrão foi

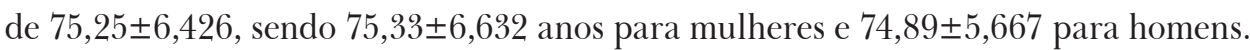

A distribuição percentual dos idosos em relação ao estado nutricional, segundo os pontos de corte do IMC estabelecido para adultos recomendada pela $\mathrm{WHO},{ }^{8}$ está representada na tabela 1. Verificou-se prevalência de sobrepeso $\left(27,48 \pm 1,284 \mathrm{Kg} / \mathrm{m}^{2}\right)$, representando $43,5 \%$ (n=20) do total da amostra. Quanto à análise estatística, não houve diferença significativa entre os sexos em relação ao sobrepeso $(\mathrm{p}=1,0000)$. No sexo feminino, o percentual de sobrepeso foi de 43,2\% $(\mathrm{n}=16)$ do total da amostra, com média de $27,58 \pm 1,212 \mathrm{Kg} / \mathrm{m}^{2}$. No masculino, o percentual de sobrepeso foi de 44,4\% (n=4), com média de 27,05 $\pm 1,689 \mathrm{Kg} / \mathrm{m}^{2}$.

Tabela 1. Distribuição percentual dos idosos de acordo com o sexo e valores de média \pm desvio padrão de acordo com a classificação do estado nutricional segundo IMC $\left(\mathrm{Kg} / \mathrm{m}^{2}\right)$ de adultos recomendada pela OMS (1997). Niterói-RJ, 2013

\begin{tabular}{ccccccc}
\hline \multirow{2}{*}{$\begin{array}{c}\text { Estado } \\
\text { Nutricional }\end{array}$} & \multicolumn{2}{c}{ Total } & \multicolumn{2}{c}{ Feminino } & \multicolumn{2}{c}{ Masculino } \\
\cline { 2 - 7 } & $\%(\mathrm{~N})$ & Média $\pm \mathrm{DP}$ & $\%(\mathrm{~N})$ & Média $\pm \mathrm{DP}$ & $\%(\mathrm{~N})$ & Média $\pm \mathrm{DP}$ \\
\hline Magreza & $6,5(3)$ & $17,12 \pm 1,020$ & 5,4 & $17,40 \pm 1,280$ & 11,1 & $16,58 \pm 0,000$ \\
& & & $(2)$ & & $(1)$ & \\
\hline Eutrofia & 26,1 & $22,74 \pm 1,394$ & 29,7 & $22,82 \pm 1,437$ & 11,1 & $21,93 \pm 0,000$ \\
& $(12)$ & & $(11)$ & & $(1)$ & \\
\hline Sobrepeso & 43,5 & $27,48 \pm 1,284$ & 43,2 & $27,58 \pm 1,212$ & 44,4 & $27,05 \pm 1,689$ \\
& $(20)$ & & $(16)$ & & $(4)$ & \\
\hline Obesidade & 17,4 & $33,01 \pm 1,313$ & 13,6 & $33,46 \pm 1,175$ & 33,3 & $32,41 \pm 1,466$ \\
Grau I & $(8)$ & & $(5)$ & & $(3)$ & \\
\hline Obesidade & 4,3 & $37,01 \pm 1,322$ & 5,4 & $37,01 \pm 1,322$ & - & \\
Grau II & $(2)$ & & $(2)$ & & & \\
\hline Obesidade & 2,2 & $40,58 \pm 0,000$ & 2,7 & $40,58 \pm 0,000$ & - & \\
Grau III & $(1)$ & & $(1)$ & & & \\
\hline
\end{tabular}

Fonte: WHO (1997). 
Em relação à circunferência abdominal, conforme apresentado na tabela 2, podemos destacar prevalência de risco elevado para doenças cardiovasculares na população do sexo feminino, representando $67,6 \%(\mathrm{n}=25)$, com média igual a 98,16 $\pm 7,601$. Já no sexo masculino, a maior parte $(n=5)$, que corresponde a 55,6\%, não apresentou risco $(91,25 \pm 1,768)$. Houve diferença estatisticamente significativa entre as mulheres e os homens que apresentaram risco moderado $(p=0,0090)$ e também entre as mulheres e os homens que apresentaram risco elevado $(p=0,0115)$.

Tabela 2. Distribuição percentual da amostra, dividida por sexo, resultados da avaliação da circunferência abdominal apresentados como média \pm desvio padrão e valores de referência para risco de doenças cardiovasculares, segundo a American Heart Association (2009). Niterói-RJ, 2013

\begin{tabular}{ccccc}
\hline \multirow{2}{*}{$\begin{array}{c}\text { Risco para doenças } \\
\text { cardiovasculares }\end{array}$} & \multicolumn{2}{c}{ Feminino } & \multicolumn{2}{c}{ Masculino } \\
\cline { 2 - 5 } & $\mathrm{N}(\%)$ & Média $\pm \mathrm{DP}$ & $\mathrm{N}(\%)$ & Média $\pm \mathrm{DP}$ \\
\hline $\begin{array}{c}\text { Normal } \\
\text { Mulheres: }<80\end{array}$ & $3(8,1)$ & $69,67 \pm 6,807$ & $5(55,6)$ & $91,25 \pm 1,768$ \\
Homens: $<94$ & & & - \\
\hline $\begin{array}{c}\text { Risco moderado } \\
\text { Mulheres: } 80-88\end{array}$ & $9(24,3)$ & $83,83 \pm 1,837$ & \\
Homens: $94-102$ & & & & \\
\hline $\begin{array}{c}\text { Risco elevado } \\
\text { Mulheres: }>88\end{array}$ & $25(67,6)$ & $98,16 \pm 7,601$ & $4(44,4)$ & $110,00 \pm 0,000$ \\
Homens: $>102$ & & & \\
\hline
\end{tabular}

Fonte: American Heart Association (2009).

Em relação à reserva proteica somática, a partir do indicador "circunferência do braço" (CB), verificou-se que, de acordo com o percentual de adequação, o sexo feminino (n=37) apresentava-se eutrófico $(96,21 \pm 16,790)$, assim como o masculino $(\mathrm{n}=9)$, com percentual de adequação médio igual a 96,21 $\pm 16,790$. Acerca da circunferência muscular do braço (CMB), o percentual de adequação do sexo feminino $(102,30 \pm 18,110)$ e do sexo masculino $(100,30 \pm 17,820)$ apresentou-se dentro dos parâmetros de eutrofia. A respeito dos valores de dobra cutânea triciptal (DCT), marcador de reserva adiposa, ambos os sexos apresentaram-se eutróficos de acordo com o percentual de

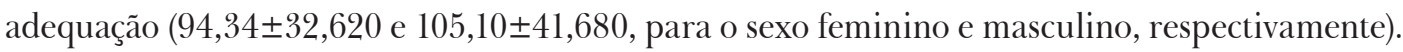
A tabela 3 apresenta os valores e percentuais de adequação da CB, DCT e CMB. 
Tabela 3. Apresentação dos valores da circunferência do braço, dobra cutânea triciptal e circunferência muscular do braço expressos por média \pm desvio padrão e seus respectivos percentuais de adequação agrupados por sexo, de acordo com a classificação de Blackburn \&Thornton (1979). Niterói-RJ, 2013

\begin{tabular}{|c|c|c|c|}
\hline & Total & Feminino & Masculino \\
\hline & Média $\pm D P$ & Média $\pm D P$ & Média $\pm D P$ \\
\hline $\mathrm{CB}(\mathrm{cm})$ & $29,28 \pm 5,030$ & $29,35 \pm 5,152$ & $29,00 \pm 4,770$ \\
\hline$\%$ de Adequação & $95,05 \pm 6,020$ & $96,21 \pm 16,790$ & $90,26 \pm 15,910$ \\
\hline Classificação & Eutrófico & Eutrófico & Eutrófico \\
\hline $\operatorname{DCT}(\mathrm{mm})$ & $18,73 \pm 7,487$ & $20,21 \pm 7,352$ & $12,83 \pm 4,783$ \\
\hline \% de Adequação & $96,50 \pm 34,370$ & $94,34 \pm 32,620$ & $105,10 \pm 41,680$ \\
\hline Classificação & Eutrófico & Eutrófico & Eutrófico \\
\hline $\mathrm{CMB}(\mathrm{mm})$ & $24,97 \pm 3,947$ & $23,54 \pm 4,150$ & $23,83 \pm 4,107$ \\
\hline$\%$ de Adequação & $91,95 \pm 14,600$ & $102,30 \pm 18,110$ & $100,30 \pm 17,820$ \\
\hline Classificação & Eutrófico & Eutrófico & Eutrófico \\
\hline
\end{tabular}

Na tabela 4, está apresentada a distribuição percentual de CB, DCT e CMB de acordo com a classificação Blackburn \& Thornton. ${ }^{12}$ A maior parte dos idosos apresentou eutrofia e não houve diferença significativa correlacionando mulheres e homens eutróficos e os não eutróficos para as variáveis CB ( $\mathrm{p}=0,4817)$, e DCT $(\mathrm{p}=0,4069)$ e CMB $(\mathrm{p}=0,1573)$. 
Tabela 4. Distribuição do percentual da circunferência do braço, dobra cutânea triciptal e circunferência muscular do braço de acordo com a classificação de Blackburn \&Thornton (1979). Niterói-RJ, 2013

\begin{tabular}{ccccccc}
\hline \multirow{2}{*}{ Classificação } & \multicolumn{5}{c}{ Feminino } & \multicolumn{3}{c}{ Masculino } \\
\cline { 2 - 6 } & $\mathrm{CB}$ & $\mathrm{DCT}$ & $\mathrm{CMB}$ & $\mathrm{CB}$ & $\mathrm{DCT}$ & CMB \\
& $\mathrm{N}(\%)$ & $\mathrm{N}(\%)$ & $\mathrm{N}(\%)$ & $\mathrm{N}(\%)$ & $\mathrm{N}(\%)$ & $\mathrm{N}(\%)$ \\
\hline $\begin{array}{c}\text { Obesidade } \\
(>120)\end{array}$ & $3(8,1)$ & $7(18,9)$ & $4(10,8)$ & - & $5(55,6)$ & - \\
\hline $\begin{array}{c}\text { Sobrepeso } \\
(120-110)\end{array}$ & $3(8,1)$ & $3(8,1)$ & $5(13,5)$ & $1(11,1)$ & - & $2(22,2)$ \\
\hline $\begin{array}{c}\text { Eutrofia } \\
(110-90)\end{array}$ & $21(56,8)$ & $11(29,7)$ & $22(59,5)$ & $4(44,4)$ & $1(11,1)$ & $3(33,3)$ \\
\hline $\begin{array}{c}\text { Desnutrição } \\
\text { leve } \\
(90-80)\end{array}$ & $6(16,2)$ & $2(5,4)$ & $5(13,5)$ & $2(22,2)$ & - & $3(33,3)$ \\
\hline $\begin{array}{c}\text { Desnutrição } \\
\text { moderada } \\
(80-70)\end{array}$ & $1(2,7)$ & $6(16,2)$ & $1(2,7)$ & $1(11,1)$ & - & \\
\hline $\begin{array}{c}\text { Desnutrição } \\
\text { grave } \\
(<70)\end{array}$ & $3(8,1)$ & $8(21,6)$ & & & \\
\hline
\end{tabular}

A distribuição do percentual de gordura corporal total apresentou-se maior no sexo feminino $(38,18 \pm 4,498 ; n=34)$ em comparação ao sexo masculino $(27,98 \pm 3,585 ; n=6)$; no entanto, ambos os sexos encontraram-se em risco de doenças associadas à obesidade, como descrito na tabela 5 . Não houve diferença estatisticamente significativa entre as mulheres e os homens que apresentaram risco de doenças associadas à obesidade $(p=0,0997)$. A estimativa do percentual de gordura corporal foi inviabilizada em 23,1\% (n=12) do total de idosos da amostra, uma vez que não foi possível realizar todas as aferições necessárias de acordo com os métodos descritos. 
Tabela 5. Distribuição do percentual de gordura corporal, expresso por média \pm desvio padrão e agrupado por sexo, segundo classificação de Lohman (1992). Niterói-RJ, 2013

\begin{tabular}{|c|c|c|c|c|}
\hline \multirow[t]{4}{*}{$\%$ de Gordura corporal } & \multicolumn{2}{|c|}{ Feminino } & \multicolumn{2}{|c|}{ Masculino } \\
\hline & \multicolumn{2}{|c|}{ Média $\pm D P$} & \multicolumn{2}{|c|}{ Média $\pm D P$} \\
\hline & \multicolumn{2}{|c|}{$38,18 \pm 4,498$} & \multicolumn{2}{|c|}{$27,98 \pm 3,585$} \\
\hline & $\mathrm{N}$ & $\%$ & $\mathrm{~N}$ & $\%$ \\
\hline $\begin{array}{c}\text { Risco de doenças associadas à } \\
\text { desnutrição }\end{array}$ & - & - & - & - \\
\hline Abaixo da média & 2 & 5,4 & - & - \\
\hline Média & - & - & - & - \\
\hline Acima da média & 2 & 5,4 & 3 & 33,3 \\
\hline $\begin{array}{l}\text { Risco de doenças associadas à } \\
\text { obesidade }\end{array}$ & 33 & 89,2 & 6 & 66,7 \\
\hline
\end{tabular}

\section{Discussão}

O presente estudo descreve o perfil antropométrico de uma amostra da população idosa atendida no Ambulatório de Nutrição do CRASI/UFF, no município de Niterói-RJ, onde a proporção de mulheres foi maior que a de homens, assim como encontrado na literatura. ${ }^{13-15}$ Isto ocorre devido à mortalidade diferencial dos sexos, onde prevalece o masculino, que perdura há vários anos no Brasil. ${ }^{16,17}$

O maior percentual de mulheres decorre, dentre outras causas, a uma menor exposição a fatores de riscos, como tabagismo e etilismo, além das diferenças de atitude entre homens e mulheres em relação ao controle e tratamento das doenças, visto que as mulheres fazem uso mais frequente de serviços de saúde. ${ }^{18}$

A amplitude da variação da idade observada no presente trabalho foi elevada e a maior idade encontrada foi de 90 anos. Este dado é relevante partindo-se do preceito de que os idosos que atingem idades mais avançadas podem apresentar redução de reservas funcionais, com aumento da vulnerabilidade a muitas doenças, as quais, consequentemente, aumentam o risco de morte. ${ }^{16}$ 
Com base no IMC, a população estudada apresentou prevalência da inadequação do estado nutricional, com destaque para o sobrepeso, assim como encontrado em Cintra et al. ${ }^{19} \mathrm{e}$ Ribeiro et al. ${ }^{15}$ Quando agrupados por sexo, os valores de IMC das mulheres são inferiores aos encontrados entre os homens, o que não corrobora outros estudos realizados com a população idosa, visto que o peso dos homens atinge o platô por volta dos 65 anos e geralmente declina, enquanto que nas mulheres este platô ocorre por volta dos 75 anos. ${ }^{14}$

Uma proporção muito menor de idosos apresentou baixo-peso, a qual é comparável a dados nacionais, que apontam que a prevalência de déficit de peso atinge 4,1\% das mulheres e 5,3\% dos homens acima de 65 anos de idade, segundo levantamento de dados realizado em todo país. ${ }^{19-20}$

Em dados mais recentes, o baixo-peso foi encontrado em pequena parcela da população idosa. A prevalência de excesso ou déficit de peso da amostra populacional avaliada, portanto, se aproxima daquela da população idosa em geral. ${ }^{19}$ Tal resultado merece ênfase, pois corrobora os dados da literatura, que, apesar dos diferentes critérios de classificação utilizados nos estudos, apontam para o desvio nutricional da população idosa. ${ }^{21}$

A classificação do estado nutricional de idosos segundo o IMC é, ainda, bastante discutida, havendo divergência, entre vários estudos, quanto aos valores empregados. ${ }^{22}$ Considerando as modificações de composição corporal no processo de envelhecimento e as evidências disponíveis até o momento, segundo Silveira et al., ${ }^{23}$ não parece adequado utilizar o mesmo ponto de corte do IMC de populações adultas para classificar obesidade em idosos. No entanto, a OMS sugere a adoção dos mesmos pontos de corte utilizados para adultos, os quais se baseiam na população norte-americana. ${ }^{24}$ Além disso, este critério foi o mesmo utilizado em inquéritos nacionais, como a Pesquisa Nacional sobre Saúde e Nutrição e a Pesquisa de Orçamento Familiar. ${ }^{23}$

Em relação ao indicador da distribuição de gordura intra-abdominal, circunferência abdominal, os resultados obtidos neste estudo demonstraram alta porcentagem de risco moderado e risco elevado para doenças cardiovasculares e distúrbios metabólicos, para ambos os sexos (67,4\%), com destaque para o sexo feminino, assim como observado no estudo de Ferreira et al. ${ }^{25}$ Também no estudo de Cintra et al. ${ }^{19}$ e Reis Filho et al., ${ }^{26}$ os valores de circunferência abdominal dos idosos revelaram elevado predomínio de indivíduos com medidas alteradas. São recentes os estudos nacionais que relacionam as medidas de circunferência abdominal às doenças crônicas entre idosos. ${ }^{20}$

Uma das hipóteses para o aumento da circunferência abdominal, sobretudo em mulheres, refere-se a algumas alterações comportamentais que são características nesta população, como a redução nos níveis de atividade física e uma alimentação desequilibrada, as quais contribuem 
também para o comprometimento do perfil antropométrico. ${ }^{27}$ Esse achado pode estar relacionado com a tendência de maior acúmulo de gordura abdominal nos indivíduos idosos. Deve-se, portanto, avaliar com cautela o acúmulo de gordura abdominal em idosos, uma vez que a redistribuição de gordura pode mascarar o diagnóstico de desnutrição, havendo aumento no depósito de gordura toraco-abdominal e diminuição da gordura subcutânea nos membros superiores e inferiores. Além disso, entre os idosos, a CAb e o IMC podem apresentar associações inversas com mortalidade. ${ }^{20}$

No que se refere à circunferência do braço, em relação ao percentual de adequação em ambos os sexos, prevaleceu a eutrofia (54,3\%), assim como os resultados obtidos nos estudos de Cabreira et al. ${ }^{28}$ que encontraram $47 \%$ dos idosos avaliados como eutróficos.

Verifica-se que os dados do presente estudo corroboram os estudos atuais, mas diferem da literatura sobre avaliação nutricional de indivíduos idosos. Esta afirma que, com o envelhecimento, observam-se redução de massa magra e aumento de gordura corporal, e nos idosos do presente estudo não foi observada tal alteração, prevalecendo o estado de eutrofia para tal parâmetro. ${ }^{28}$

No parâmetro DCT, os idosos de ambos os sexos apresentavam-se eutróficos; as mulheres, no entanto, apresentaram valores maiores $(20,21 \pm 7,352)$ quando comparadas aos homens $(12,83 \pm 4,783)$, sem diferença estatisticamente significativa. Os valores de DCT são congruentes com as informações disponíveis na literatura, que indicam maior acúmulo de gordura nas extremidades femininas, quando comparadas às masculinas. ${ }^{14}$

Estudos mostram que os homens tendem a ter maiores depósitos de massa magra em relação às mulheres; no entanto, no presente estudo, ambos os sexos apresentaram resultados semelhantes

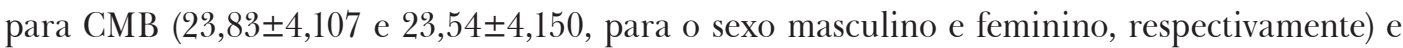
não houve diferença estatisticamente significativa quando comparados os sexos e o percentual de adequação $(\mathrm{p}=0,1573){ }^{14}$

A avaliação da composição corporal dos idosos é muito importante, pois alterações na distribuição de gordura corporal podem estar associadas a desordens metabólicas e cardiovasculares. ${ }^{29}$

Segundo Moreira et al., ${ }^{27}$ o somatório de dobras cutâneas é considerado um excelente indicador para estimar a gordura subcutânea, por ser previamente validado para a população brasileira. Os resultados deste estudo indicam maior acúmulo de gordura subcutânea nas mulheres em relação aos homens, assim como encontrado no estudo de Reis Filho e colaboradores ${ }^{26}$ e em outros estudos no Brasil e no mundo. ${ }^{29}$ 


\section{Conclusão}

A partir do presente estudo, pode-se concluir que há incidência de inadequações do estado nutricional dos idosos atendidos no Ambulatório de Nutrição do Centro de Referência em Assistência à Saúde do Idoso (CRASI) da Universidade Federal Fluminense (UFF), no município de Niterói-RJ, reveladas pelo excesso de peso de acordo com o IMC e pela gordura abdominal aumentada, que indicam a maior susceptibilidade desses idosos às DCNTs, influenciando o aumento da morbimortalidade.

Os resultados observados sugerem atenção quanto à importância de intervir nutricionalmente na rotina dessa população, para que estratégias alimentares possam auxiliar na diminuição dos valores antropométricos elevados, colaborando para a redução de riscos de doenças associadas às inadequações no estado nutricional.

\section{Referências}

1. Chehuen Neto JA, Sirimarco MT, Cândido TC, Barboza DF, Gonçalves ECQ, Gonçalves RT. Perfil epidemiológico dos idosos institucionalizados em Juiz de Fora. HU Rev. 2011; 37(2):207-16.

2. Silva HO, Carvalho MJAD, Lima FEL, Rodrigues LV. Perfil epidemiológico de idosos frequentadores de grupos de convivência no município de Iguatu, Ceará. Rev. Bras. Geriatr. Gerontol. 2011; 14(1):12333.

3. Instituto Brasileiro de Geografia e Estatística. Pesquisa nacional por amostra de maio 2015]. Disponível em: http://www.ibge.gov.br/home/estatistica/populacao/trabalhoerendimento/ pnad2009/pnad_sintese_2009.pdf

4. Panziera FB, Dorneles MM, Durgante PC. Avaliação da ingestão de minerais antioxidantes em idosos. Rev. Bras. Geriatr. Gerontol. 2011; 14(1):49-58.

5. Freitas AMP, Philippi ST, Ribeiro SML. Listas de alimentos relacionadas ao consumo alimentar de um grupo de idosos: análises e perspectivas. Rev. Bras. Epidemiol. 2011; 14(1):161-77.

6. Carvalho JA, Carvalho, AP, Alves FA. Perfil nutricional associado ao índice de obesidade de idosos do Centro de Saúde Sebastião Pinheiro Bastos, AAP - VR, Volta Redonda, RJ. Rev. Práxis. 2009; $1(1): 43: 50$.

7. International Society for the Advancement of Kinanthropometry. International standards for anthropometric assessment. Underdale: ISAK; 2006.

8. World Health Organization. Defining the problem of overweight and obesity. In: World Health Organization. Obesity: preventing and managing the global epidemic: report of a Who Consultation. Geneva: 2000. WHO technical report series, 894.

9. Alberti KGMM, Ecke RH, Grundy SM, Zimmet PZ, Cleeman JI, Donato KA, et al. Harmonizing the metabolic syndrome. Circulation 2009; 120:1640-5. 
10. Durnin JVGA, Womersley J. Body fat assessed from total body density and its estimation from skinfold thickness: measurement on 481 men and women aged from 16 to 72 years. Br. J. Nutr. 1974; 32(1):77-97.

11. World Health Organization. Obesity: preventing and managing the global epidemic of obesity. Report of the WHO consultation of obesity. Gevena: WHO; 1997.

12. Blackburn GL, Thornton PA. Nutritional assessment of the hospitalized patient. Med Clin North Am. 1979; 63:11103-11115

13. Mastroeni MF, Mastroeni SSBS, Erzinger GS, Marucci MFN. Antropometria de idosos residentes no município de Joinville-SC, Brasil. Rev. Bras. Geriatr. Gerontol. 2010; 13(1):29-40.

14. Menezes TN, Marucci MFN. Avaliação antropométrica de idosos residentes em Instituições de Longa Permanência de Fortaleza-CE. Rev. Bras. Geriatr. Gerontol. 2010; 13(2):235-43.

15. Ribeiro RL, Tonini KAD, Presta FMP, Souza MVM, Picanço EA. Avaliação nutricional de idosos residentes e não residentes em Instituições geriátricas no Município de Duque de Caxias, RJ. Revista Eletrônica Novo Enfoque 2011; 12(12):39-46.

16. Gontijo EEL, Silva MG, Lourenço AFE, Inocente NJ. A população idosa de Gurupi: avaliação do perfil sociodemográfico, de saúde e do uso de medicamentos. Revista Científica do ITPAC 2013; 6(1). Disponível em: http://www.itpac.br/arquivos/Revista/61/1.pdf

17. Iwamoto C, Silva RB, Santos LC, Coutinho VF. Estado nutricional, qualidade de vida e doenças associadas em idosos residentes em instituições de longa permanência. Geriatria \& Gerontologia. 2008; 2(2):42-8.

18. Garcia ESS, Saintrain MVL. Perfil epidemiológico de uma população idosa atendida pelo Programa Saúde da Família. Rev. Enferm. UERJ 2009; 17(1):18-23.

19. Cintra RMG, Oliveira D, Silva LMG. Estado nutricional e ocorrência de hipertensão arterial e de diabetes em idosos residentes e não residentes em instituições geriátricas. Alimentos e Nutrição Araraquara. 2012; 23(4):567-75

20. Nascimento CM, Ribeiro AQ, Sant'Ana LFR, Oliveira RMS, Franceschini SCC, Priore SE. Estado nutricional e condições de saúde da população idosa brasileira: revisão da literatura. Rev. Assoc. Méd. Minas Gerais. 2011; 21(2):174-80.

21. Silva AKQ, Gusmão SC, Castro KR, Moreira RAN, Morais AHA. Perfil nutricional de idosos assistidos em instituição de longa permanência na cidade de Natal, RN. Geriatria \& Gerontologia. 2010; 4(1):27-35.

22. Bassler TC, LEI DLM. Diagnóstico e monitoramento da situação nutricional da população idosa em município da região metropolitana de Curitiba (PR). Rev. Nutr. 2008; 21(3): 311-21.

23. Silveira EA, Gilberto KAC, Barbosa LS. Prevalência e fatores associados à obesidade em idosos residentes em Pelotas, Rio Grande do Sul, Brasil: classificação da obesidade segundo dois pontos de corte do índice de massa corporal. Cad Saude Publica. 2009; 25(7): 1569-77.

24. Rauen MS, Moreira EAM, Calvo MCM, Lobo AS. Avaliação do estado nutricional de idosos institucionalizados. Rev. Nutr. 2008; 21(3):303-10. 
25. Ferreira CCC, Peixoto MRG, Barbosa MA Silveira EA. Prevalência de fatores de risco cardiovascular em idosos usuários do Sistema Único de Saúde de Goiânia. Arq. bras. cardiol. 2010; 95(5):621-8.

26. Reis Filho ADR, Coelho CF, Voltarelli FA, Ferrari Junior J, Ravagnani FCP, Fett WCR et al. Associação entre variáveis antropométricas, perfil glicêmico e lipídico em mulheres idosas. Rev. Bras. Geriatr. Gerontol. 2011; 14(4):675-86.

27. Moreira AJ, Nicastro H, Cordeiro RC, Coimbra P, Fragella VS. Composição corporal de idosos segundo a antropometria. Rev. bras. geriatr.gerontol. 2009; 12(2):201-13.

28. Cabreira TP, Marcuzzo ML, Kirsten VR. Perfil nutricional de idosos de uma instituição geriátrica de Santa Maria-RS. Disc. Scientia. Série: Ciências da Saúde. 2008; 9(1):69-76.

29. Machado RSP, Coelho MASC, Coelho KSC. Percentual de gordura corporal em idosos: comparação entre os métodos de estimativa pela área adiposa do braço, pela dobra cutânea tricipital e por bioimpedância tetrapolar. Rev. bras. geriatr. gerontol. 2010; 13(1):17-27.

Recebido: 04/4/2015

Revisado: $10 / 4 / 2015$

Aprovado: 30/4/2015 